\title{
Application of location detection system using active type RFID tags to railways
}

\author{
K. Seki, S. Suzuki, M. Ukai \& R. Tsuchiya \\ Railway Technical Research Institute, Japan
}

\begin{abstract}
As active type RFID tags can transmit their identification information about $10 \mathrm{~m}$ by radio, we can calculate locations of persons who hold tags with an accuracy of a few meters, by installing tag receivers appropriately and by the function of a location calculating server which analyzes the gathered information regarding receivers which have received the tags' identification information. We are developing two application systems to make use of active type RFID tags. The first one is an information provision system for passengers. In this system, passengers hold tags, location calculating servers are installed at stations and in trains, and an information delivering server is located at a management center. The location calculating servers at stations always transmit calculated location information of tags to information delivering server via an Intranet. On the other hand, the location calculating servers in trains store the calculated location information while trains are running between stations and transmit them at a stroke via communications lines such as wireless Local Area Networks to the information delivering server when the trains stop at stations. Therefore, the information delivering server can trace the locations of passengers continuously and manage their status such as entering into stations and getting off trains almost on time. Accordingly, it can deliver guidance information to mobile phones of the passengers via e-mail at appropriate places and timing. The second one is a system to cope with abnormal situations. In this system, station staffs hold tags. When a video surveillance system detects an illegal event such as violence and suspicious loitering, it transmits an alarm to the nearest staff to directly go to the location. As the locations of the staff are detected by tags indoors and by Global Positioning System (GPS) outdoors, they can be continuously traced wherever they are.
\end{abstract}

Keywords: active type RFID tag, Information provision system, video surveillance system. 


\section{Introduction}

In the future ubiquitous society, grasping locations of persons is one of the most essential things in order to understand their located situations and provide the most suitable services to them. Locations information of railway workers is also important to improve efficiency and rapidness of daily works. Grasping their positions at any places and tracing their movement continuously, however, have not been realized yet, because in a railway system there are so many varieties of environments where locations of persons are to be detected such as indoors, outdoors, and on-board. Though a lot of technologies to detect locations are proposed and realized, it is impossible for only one method to cover all places and requirements.

In this paper, we focus on active type RFID tags and propose two application systems. First we survey a few technologies to detect locations of persons and compare their characteristics. We then explain the first application system, which detects locations of passengers and provides appropriate information to them according to their locations. We tentatively produced this system and verified that the system worked well. Next, we explain the second application system, which grasps locations of station staffs by both active type RFID tags and GPS, detects some abnormal events, and alarms the staffs nearest to the detected event. Finally, we summarize the discussion as the conclusion.

\section{Location detection of persons in railways}

In this chapter, we survey examples of technologies to detect locations of persons in railways. As these technologies have their own merits and demerits, it is thought to be effective to develop an appropriate system by combining these technologies.

\subsection{Global Positioning System (GPS)}

GPS is available outdoors with the precisions of about $15 \mathrm{~m}$, and commercial services to guide persons who hold cellular phones with GPS receivers are emerging. However, GPS is difficult to use indoors and on-board, and the precision may be worse or even the location detection itself is impossible when positions of satellites are inappropriate.

\subsection{Entering and exiting data of Automatic Fare Collection (AFC) gates}

By using entering and exiting data that AFC gates record, it is possible to grasp entering or exiting a station of a passenger without any action by a passenger except inserting or touching a ticket to a gate. Passenger guidance system [1] is an example that utilizes this method. This experimental system is a partial realization of CyberRail, which is an information technology based infrastructure of system concept we are proposing. The system informs appropriate information to passengers via e-mails over cellular phones according to their 
travel schedules and current locations. Their locations between starting stations and destination ones are estimated based on their travel schedules and train diagrams. Many users highly rated the service provided by the system, but some people pointed out that guidance at complicated stations is needed.

\subsection{Radio Frequency Identification (RFID) tags}

There are a lot of varieties of RFID tags, which are different in ranges of communications with receivers and information rates transmitted.

Passive type RFID tags that do not contain batteries can communicate in a range from about a few centimeters through a few ten centimeters. So if a tag is detected by a receiver, it is certain that the tag passes the position where the receiver exists. On the other hand, actions to make the tag close to the receiver are required. Guidance system for handicapped people [2] is an example to utilize passive type RFID tags. Passive type RFID tags are embedded under a Braille block. In a guidance system for visually impaired people, for example, a white cane receives location data from an RFID tag by using an antenna installed in its top, and transmits them to a portable terminal. The portable terminal provides appropriate information to the user.

As active type RFID tags that contain batteries can communicate in a range of a few ten meters, they are effective in cases where the precision of location is required to be about a few meters without any action of users. So this technology was used by an experimental information provision system at shopping mall.

\section{Information provision system for passengers}

If locations of passengers can be detected at such precision as "they are around a gate" or "they are in a car No.1," a lot of satisfiable functions of information provision systems will be provided to passengers. As active type RFID tags are considered to be suitable for this precision, we tentatively produced an information provision system by improving the above-mentioned experimental information delivering system for shopping streets.

\subsection{Overview of information provision system}

The information provision system consists of active type RFID tags, receivers, collection units, location servers, and information delivering servers.

\subsubsection{Active type RFID tags}

Figure 1(a) shows an active type RFID tag which a passenger receiving guidance information holds. A transmission frequency is $314.950 \mathrm{M} \mathrm{Hz}$ and a communications distance is about $20 \mathrm{~m}$. The tag transmits its identification information of 16 bits length every 1 through $7 \mathrm{~s}$. Its size is about $4 \mathrm{~cm} \mathrm{x} 3 \mathrm{~cm}$. 


\subsubsection{Receiver}

Figure 1(b) shows a receiver. A receiver receives identification information which active type RFID tags transmit. A receiver has two antennas, one is used to receive identification information, and another is to transmit the receiving identification information of active type RFID tags together with its own identification information to a collection unit via wireless networks dynamically configured among receivers.

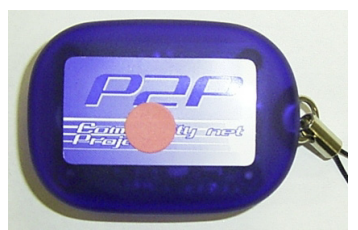

(a)

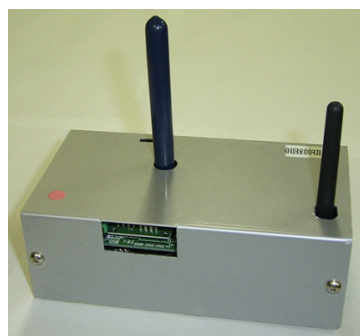

(b)

Figure 1: (a) Active type RFID tag and (b) Receiver.

\subsubsection{Collection unit}

A collection unit gathers identification information of both active type RFID tags and receivers transmitted from all receivers, and transmits them to a location server via a serial port. An appearance of a collection unit is the same as that of a receiver except that it has only one antenna.

\subsubsection{Location server}

A location server identifies locations of active type RFID tags by calculating mean values of coordinates of receivers which receive the identification information of the tags, and notices the locations information to an information delivering server. Figure 2 illustrates an example of a measurement when a person with an active type RFID tag, whose transmission period was $3 \mathrm{~s}$, walked straight at a speed of $1 \mathrm{~m} / \mathrm{s}$ between the two parallel lines, on which receivers, whose sensitivity was set at about $10 \mathrm{~m}$, were located every $10 \mathrm{~m}$ on two lines. Black circles in Fig. 2 are calculated locations by active type RFID tags, and white circles connected with black circles by segments are corresponding real locations.

\subsubsection{Information delivering server}

An information delivering server traces locations of passengers and sends appropriate e-mails according to their profiles to cellular phones of the passengers at appropriate time. 


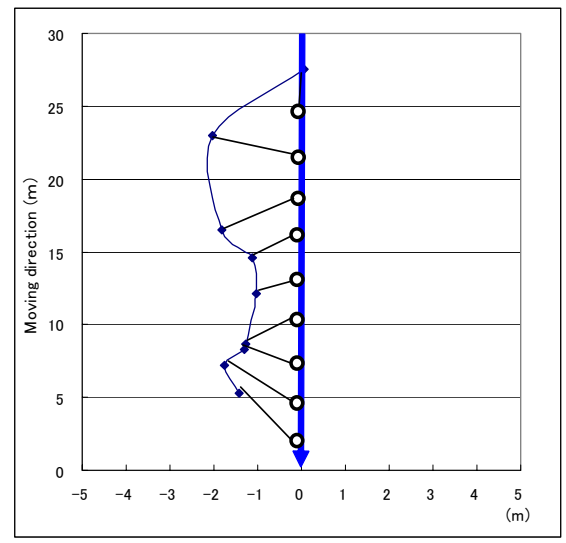

Figure 2: Example of precision of locations by active type RFID tag.

\subsection{Items improved from the system for shopping street}

The information provision system for passengers and the information delivering system for shopping streets are the same in the sense that both systems deliver information according to current locations and profiles of users. The information provision system for passengers has to take into accounts the following matters or requirements.

(1) Users of the system move not only in geographically continuous area but also across heterogeneous environments such as from a station to a train. Moreover, trains themselves which are environments for the user move.

(2) As it is not always possible to communicate between trains and on-ground systems continuously, locations of on-board users are not always grasped.

(3) It is necessary to take into consideration absolute locations of users as well as relative locations between users and trains. It is also necessary to judge situations such as "in a station" and "on-board" and histories of changes of the situations of users.

(4) Ticketing information is required as a part of users' profile information.

To cope with the above matters or requirements, we have improved location servers and information delivering servers as follows.

\subsubsection{Location server}

It is necessary to trace locations of on-board passengers in order to detect that they get on trains or to notify them of their mistakes if they are wrong vehicles. It is also necessary to define on-board coordinate system apart from coordinates system of stations, and it is desirable to define a coordinate system of each station separately. So we have developed on-board location servers and stations' location servers, which independently calculate locations of active type RFID tags. Stations' location servers have the same functions as location servers of the 
experimental information delivering system for shopping streets. On-board location servers store calculated locations information of on-board active type RFID tags if communications links between trains and wayside base stations are disconnected, and transmit the stored information to an information delivering server in the lump when the communications links are established. As a result we can continuously gather locations information of passengers and manage histories of their movement including on-board, though some time delays occur in grasping the location information of on-board passengers.

Receivers are also installed appropriately both on-board and in stations. Active type RFID tags are also installed on-board to make stations' location server detect their locations.

\subsubsection{Information delivering server}

We have provided two basic guidance functions, namely, "guidance on a platform before getting on a train" and "guidance on a platform after getting off a train." An information delivering server has to trace status of passengers in order to deliver necessary guidance information. We have defined three states in this experimental system, namely, "Initial (Outside of railway premise)", "In a station", and "On-board." Figure 3 is a state transition diagram. An information delivering server determines timings of information delivery according to both state transitions of passengers and locations of trains.

We also provided a management function to input and update information about trains such as identification information of active type RFID tags installed on the trains and types of the trains, and profile of passengers such as identification information of active type RFID tags, tickets they hold and their e-mail addresses.

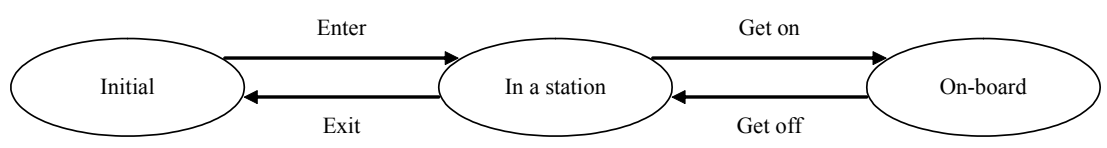

Figure 3: State transition diagram.

\subsection{Experiments in compound of RTRI}

We carried out experiments to verify functions of our experimental system with a train and mock stations.

\subsubsection{Configuration of experiments}

Figure 4 shows configurations of experiments. There are two mock stations, namely Station A and Station B, and Local Area Networks (LAN) of the stations are connected by Wireless LAN (WLAN). When a train reaches one of the stations, a communications link between the train and an access point of WLAN of the station is established. There are location servers and receivers at the stations and in the train. An information delivering server is connected to the LAN of Station A. 


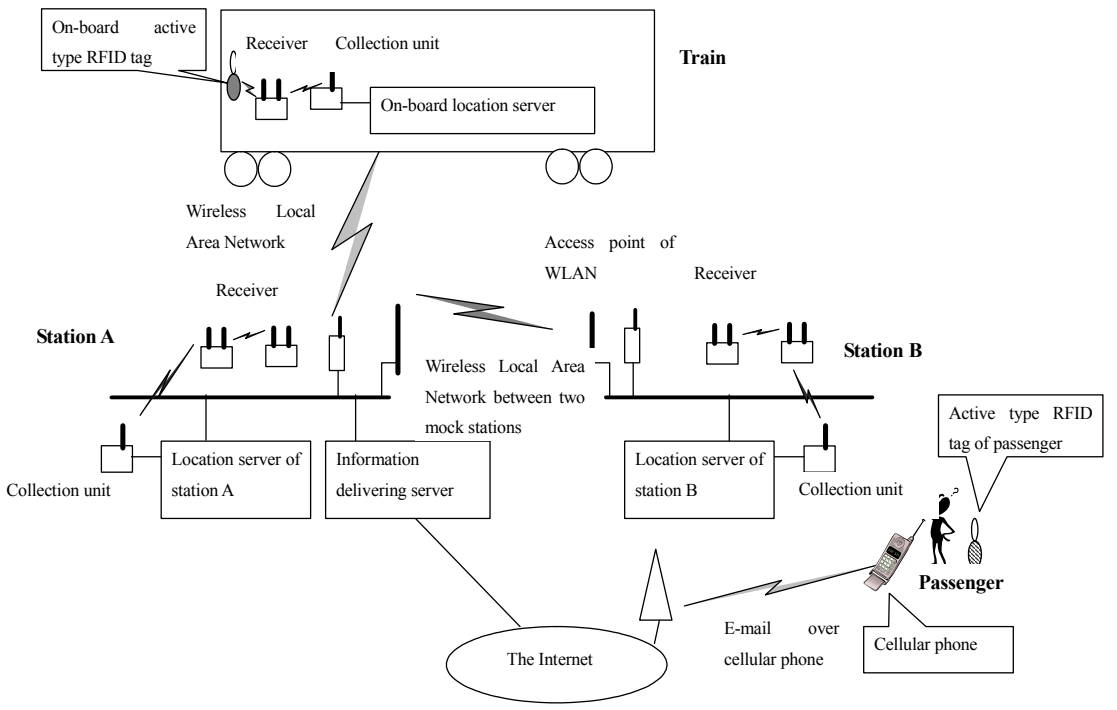

Figure 4: Configurations of experiments.

\subsubsection{Scenarios of experiments}

We verified operations of the system when users with active type RFID tags and cellular phones moved in the following scenarios.

(1) A passenger gets on the train at Station A and gets off at Station B. There are two sub-cases, that is, the passenger comes to Station A faster than the train and vise versa. We verified that "guidance on a platform before getting on a train" and "guidance on a platform after getting off a train" were performed at Station A and Station B, respectively.

(2) A passenger gets on the train at Station A and the train moves to Station B. The passenger gets off the train and gets on the train again at Station $\mathrm{B}$, and the train comes back to Station A, and finally the passenger gets off the train. This simulates the movement of the passenger from Station A to another station via Station B. We verified that "guidance on a platform before getting on a train" was performed when the passenger got on the train at Station A and "guidance on a platform after getting off a train" was performed when the passenger got off the train at Station A, as well as no guidance was performed at Station B.

(3) There are two passengers $P$ and $Q$ at Station A. P has a ticket for the train $\mathrm{X}$ and $\mathrm{Q}$ has a ticket for the train $\mathrm{Y}$. We verified that "guidance on a platform before getting on a train" was performed to $\mathrm{P}$ but not to $\mathrm{Q}$ when train $\mathrm{X}$ arrived at the station.

There was almost no delay between the time when the information delivering server transmitted e-mail and the time when the cellular phone received the e-mail. There is possibility of delayed or even undelivered emails, however, as the system cannot control transmission qualities for the Internet and cellular networks. 


\subsection{Extensions of the experimental system}

We extended functions of the experimental system to verify that it could comply with more complicated situations.

\subsubsection{Supposed situation}

We supposed the situations in which a train was composed of different types of cars. Figure 5 shows an example of such situations. For passengers who get on a train which splits at an intermediate station, guidance information shall be provided according to their destination stations and current locations. We assume that a passenger $\mathrm{P}$ travels from station $\mathrm{A}$ to Station $\mathrm{D}$ and a passenger $\mathrm{Q}$ from station A to station $\mathrm{C}$.

(1) At Station A: P shall be guided "Please go to the platform and stay near Car No.1," but Q may be guided "Please go to the Platform."

(2) On-board: P shall be guided "Please move to Car No.1" if he/she is in Car No.2.

(3) At Station B: Q shall be guided "You must get off at the next station."

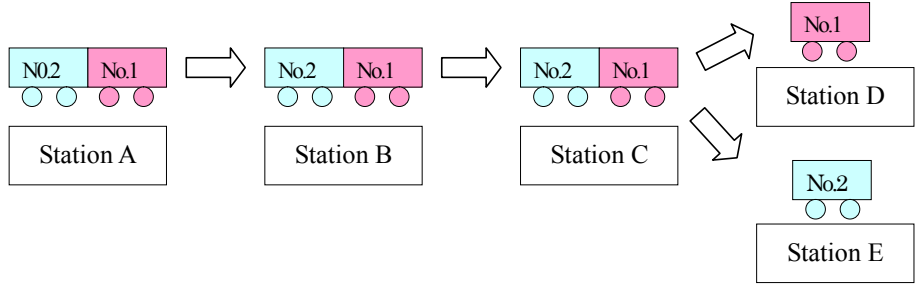

Figure 5: Split train.

\subsubsection{Modifications of information delivering server}

To cope with the above situations, we modified programs of information delivering server.

(1) A state "In a station" was divided to two states, "At a gate" and "On a platform."

(2) Trains information was extended to include their compositions.

We verified that this system could flexibly cope with sub-divisions of states and additions of contents of guidance.

\section{Abnormal situations detection and notification system for railway staff}

When alarms are transmitted to the railway staff nearest to the location where any accident happens, required precision of locations of the staffs is about a few meters, and active type RFID tags are suitable. On the other hand, staffs move around not only in station buildings but also outdoors, where receivers of the tags are difficult to install. So it is necessary to use other methods to detect locations. As GPS is such a popular method, we are considering combinations of active type RFID tags and GPS. 


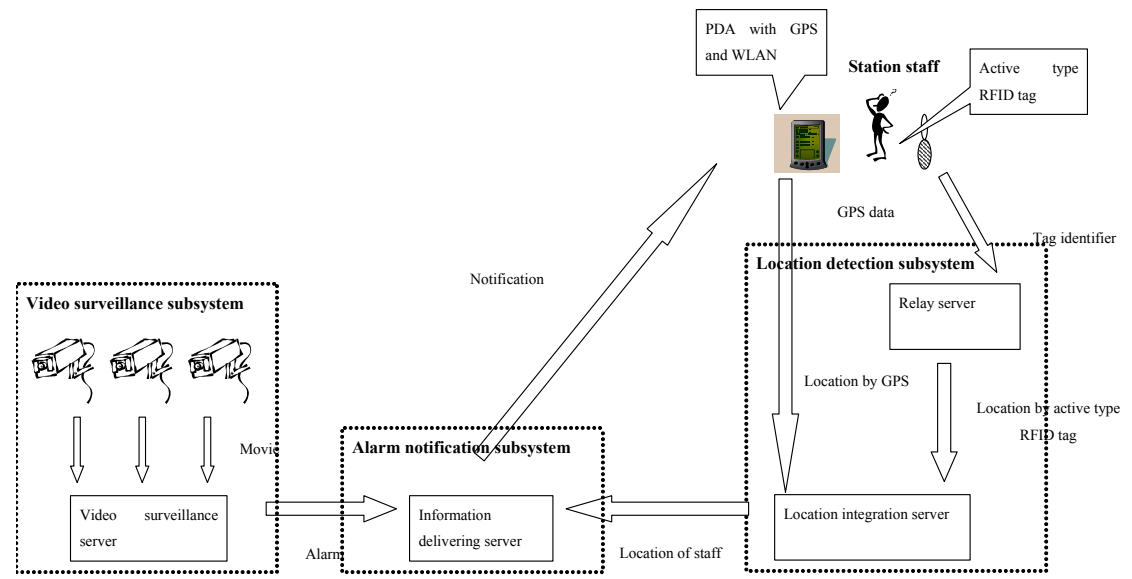

Figure 6: Outline of emergency detection and notification system.

Figure 6 shows an outline of an abnormal situations detection and notification system. This system consists of three subsystems, that is, a video surveillance subsystem, location detection subsystem, and alarm notification subsystem. A station staff holds an active type RFID tag and a Personal Digital Assistance (PDA) with GPS receiver and WLAN interface.

\subsection{Video surveillance subsystem}

This subsystem consists of video cameras and a video surveillance server. The server detects abnormal situations based on movies transmitted from the video cameras processed by image processing technology, and transmits an alarm which includes a type of the event and occurrence time of the event to an alarm notification subsystem. Examples of the types of events are people gathering, suspicious loitering, left luggage, people fighting, and people fall.

\subsection{Location detection subsystem}

This subsystem consists of a relay server and a location integration server. The relay server, which is installed at each floor of a station, gathers identification information of active type RFID tags and transmits them to a location gathering server. A PDA periodically calculates its location by its GPS receiver and transmits the result to the location integration server via WLAN. The location integration server finally calculates locations of the PDA from location information by an active type RFID tag and GPS, and transmits the result to an alarm notification subsystem.

\subsection{Alarm notification subsystem}

This subsystem consists of an alarm notification server. When an alarm notification server receives an alarm from the video surveillance subsystem, it 
determines station staffs to whom notifications should be sent considering their current locations, and transmits them via WLAN.

We are now developing the prototype system and will carry out some experiments in our Research Institute. (This work is financially supported in part by the Japanese Ministry of Land, Infrastructure and Transport.)

\section{Conclusion}

Locations information of persons are essential for ubiquitous services in railways. In this paper, we have proposed two systems, one is to provide information to passengers and another is to detect and alarm abnormal events, both of which utilize locations information detected by active type RFID tags. The information provision system was experimentally manufactured and verified to provide appropriate guidance to passengers.

Next, we will manufacture experimentally the abnormal events detection and notification system and evaluate the effectiveness of the system.

\section{References}

[1] Ogino, T., Tsuchiya, R. : Realization of CyberRail Partial Functions in Actual Environment, Quarterly Report of RTRI, pp.18-22, Vol.46, No.1, 2005

[2] Matsubara, H., Fukazawa, N., Myojo, S., Tsuchiya, R. : Guidance System for Handicapped People, Quarterly Report of RTRI, pp.13-17, Vol.46, No.1, 2005 